\title{
Voltage Controlled Intertwined Spiral Arrays for Reconfigurable Metasurfaces
}

\author{
A. Vallecchi, ${ }^{1}$ R. J. Langley, ${ }^{1}$ and A. G. Schuchinsky ${ }^{2}$ \\ ${ }^{1}$ Department of Electronics and Electrical Engineering, University of Sheffield, Sheffield S1 3JD, UK \\ ${ }^{2}$ Institute of Electronics, Communications and Information Technology, Queen's University of Belfast, Belfast BT3 9DT, UK \\ Correspondence should be addressed to A. Vallecchi; a.vallecchi@sheffield.ac.uk
}

Received 2 December 2013; Accepted 9 February 2014; Published 20 March 2014

Academic Editor: Douglas H. Werner

Copyright (c) 2014 A. Vallecchi et al. This is an open access article distributed under the Creative Commons Attribution License, which permits unrestricted use, distribution, and reproduction in any medium, provided the original work is properly cited.

\begin{abstract}
Reconfigurable bistate metasurfaces composed of interwoven spiral arrays with embedded pin diodes are proposed for single and dual polarisation operation. The switching capability is enabled by pin diodes that change the array response between transmission and reflection modes at the specified frequencies. The spiral conductors forming the metasurface also supply the dc bias for controlling pin diodes, thus avoiding the need of additional bias circuitry that can cause parasitic interference and affect the metasurface response. The simulation results show that proposed active metasurfaces exhibit good isolation between transmission and reflection states, while retaining excellent angular and polarisation stability with the large fractional bandwidth (FBW) inherent to the original passive arrays.
\end{abstract}

\section{Introduction}

Planar metamaterials formed by a single layer of electrically small scatterers arranged into two-dimensional periodic arrays have recently attracted increasing interest owing to the ease of fabrication and the intricate properties appealing for potential applications. Metasurfaces, as planar metamaterials are usually called, allow, for example, for effective control of polarization, frequency selectivity, and multiband operation while being deployed on conformal surfaces; see for example, recent review [1]. They can also be used in ultrathin absorbers of substantially sub-wavelength thickness [2-4], to implement slow-light effects in compact configurations [5], and designed to create mantle cloaking devices capable of drastically suppressing the scattering from both planar and 3D objects [6]. The distinctive feature of metasurfaces is the sub-wavelength size of their unit cells, which provides the advantages of angular invariance of the array response and suppression of the higher order diffraction effects.

Miniaturization of periodic structures is an important prerequisite for their integration in small mobile terminals, RF front-ends, conformal frequency selective surfaces (FSSs), and other applications where one of the main challenges is that a large number of unit cells are usually required for the array to effectively interact with an incident field [7]. Moreover, the small electrical size of the unit cells plays the pivotal role in the angular and polarisation stability of the array response to the incident fields. Both of these aspects are essential for FSS applications to wireless communication systems operated in the controlled electromagnetic architecture of buildings with enhanced spectral efficiency and security. In fact, on the one hand, the wavelengths in the frequency bands used in indoor communications can be comparable with the size of building interiors and ordinary office rooms. On the other hand, the propagation characteristics of the built environment can be complex due to diffraction, reflection, scattering, and multipath propagation, as demonstrated in [8] by the finite-difference time-domain simulations of electromagnetic wave propagation in buildings. Thus it can be argued that only FSSs with a very stable frequency response over a wide range of incidence angles are suitable for walls controlling interference and shielding in the built environment.

Among the many different types of layouts that have been proposed and used in the design of FSSs, periodic arrays of convoluted and interwoven elements have demonstrated 
many unique and attractive properties [9-14]. The main distinctive feature of such arrays is that their fundamental resonance occurs at substantially sub-wavelength size of the unit cell, thus qualifying them as metasurfaces. In addition, the interleaved conductors extended into adjacent unit cells broaden fractional bandwidth (FBW) of the fundamental resonance. For example, the free-standing intertwined quadrifilar spiral arrays were shown to achieve the $-10 \mathrm{~dB}$ FBW of $\sim 55 \%$ at the unit cell size $\sim 1 / 40$ th of the resonance wavelength [13]. The latter property of the interwoven patterns is in stark contrast to the conventional periodic structures with the constituent elements confined to a single unit cell of the size commensurate with half a resonance wavelength, where FBW rapidly narrows as the resonance frequency lowers $[15,16]$.

The use of FSS and metasurfaces for efficient control of the electromagnetic environment of buildings faces additional challenges. Namely, passive FSSs, although suitable for many fixed frequency applications, become restrictive in the buildings with diverse spectral requirements and propagation conditions changeable due to the physical movement of dividing walls or partitions. This implies that a FSS needs the capability of adjusting its response in accordance with the variable architectural requirements. Active FSSs (AFSSs) with embedded pin diodes serve to this purpose by switching between the transmission and reflection modes [17].

In this paper, we demonstrate that the interwoven spiral arrays with periodic and tessellated conductor patterns proposed in $[12,13,18]$ are particularly apt for realization of bi-state switchable AFSSs with integrated voltage control circuitry. Initially, the concept of the switch-mode AFSS is elucidated using an example of entwined bifilar spirals with embedded pin diodes. In this arrangement, the AFSS exhibits a voltage-controlled band-stop response to one polarisation of the incident field while remaining always transparent to the orthogonal polarisation. This approach is then extended to the AFSSs responsive to both TE and TM polarisations of the incident waves.

The rest of the paper is organised as follows. The concept of the proposed AFSS is described and elucidated in Section 2 using an example of intertwined bifilar spiral AFSS. The effects of the pin diode parameters in the on and off states and the unit cell layout on the AFSS performance are discussed here for single polarisation of the incident field. The dual polarised bistate AFSSs composed of intertwined quadrifilar spirals are presented in Section 3 where their performance is discussed in detail for the normal and oblique incidence of TE and TM waves. The main findings and obtained results are summarised in Section 4.

\section{Anisotropic Bistate Interwoven Spiral Metasurface}

2.1. Topology and Operational Principle of Bifilar Spiral AFSS. Semiconductor switches, such as pin diodes, are commonly used to realize bistate switching of AFSSs comprised of dipoles and other element types; see for example, [17, 1924]. The term bistate means that the FSS can be made either reflective or transparent at the frequency of interest by switching on and off a control signal, usually a dc bias. The major challenge in the implementation of such AFSSs is related to delivery of the dc control signal throughout the AFSS without distorting its RF performance.

In a bistate dipole FSS, the ends of adjacent dipoles are connected by pin diodes to form columns that are linked together with biasing lines. The surface behaves as a conventional reflective array when the diodes are off, whereas when the diodes are in the on state, the array columns behave as continuous conducting strips presenting inductive impedance and acting as a high-pass filter. If the high-pass band is low enough in frequency, the surface can be substantially transparent to the incident waves $[19,20]$.

A different approach, similar to that applied to annular ring FSS in [24], is explored in this work for the implementation of bistate voltage-controlled interwoven spiral arrays. In particular, we initially focus on FSSs formed by interwoven bifilar spiral elements. These elements are conceptually similar to the original interwoven quadrifilar spiral layout presented in $[12,13]$. But since only two spiral conductor arms extend from the adjacent unit cells into a reference unit cell and are counter-wound in the gaps between the turns of the primary reference spiral, they form either column- or rowwise patterns depending on whether the number of spiral folds is even or odd. As a result, these surfaces are nearly transparent to one of the incident field polarisations, whereas they exhibit a strong resonant response to the orthogonal polarisation. Similarly to the interwoven quadrifilar spirals, the fundamental resonance of interwoven bifilar spiral FSS has broad FBW and occurs at wavelengths large as compared to the unit cell size.

To enable the desired reconfigurability of this type of AFSS, gaps are introduced in the arms of the bifilar spirals first with the aim of suppressing the fundamental resonance by such discontinuities. The strip breaks can be positioned, for example, at the periphery of each unit cell, as shown in Figure 1, but alternative locations and a different number of breaks can be also used. Then pin diodes are inserted across the strip breaks. When the diodes are in the off state, the AFSS is transparent because it is no longer resonant at its original design frequency. On the contrary, switching the diodes in the on state reconnects the spiral arm segments and the AFSS provides its original stop-band response. In the transparent state, the AFSS still exhibits resonance, which however can be shifted at other frequency depending on the number of breaks and switches introduced in the spiral arms, in order to achieve the required level of transparency in the operational band.

In this arrangement, spiral arms themselves act as current carrying conductors supplying the dc bias for switching the pin diodes. This way there is no need in external bias lines that can cause multiple resonances and interference which may drastically affect the FSS response at oblique incidence. The continuity of dc path is realized by means of suitably chosen inductors which are connected between the open ends of interwoven spiral arms while being isolated from the pair of spiral arms connected at the centre of the reference unit cell. Inductors present high impedance at the design frequency and work as RF chokes, to ensure the isolation between 


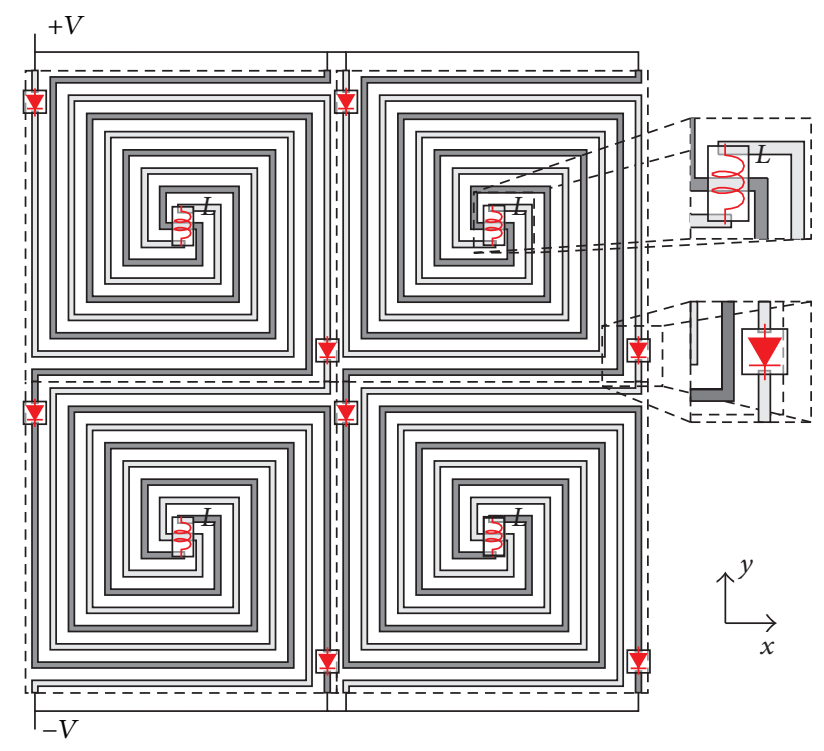

FIGURE 1: Layout of $2 \times 2$ array portion of sample free-standing bistate interwoven 9-fold bifilar spiral elements. The reference basic spirals in adjacent unit cells are alternatively marked dark and light grey to highlight the intertwining scheme. Two pin diodes are connected across breaks in the outer folds of the spiral conductors and the choke inductance is placed at the centre of each unit cell between the ends of disconnected spiral arms. Geometrical parameters of the unit cell: lattice period $p=10.2 \mathrm{~mm}$, spiral pitch $2.4 \mathrm{~mm}$, and strip width $s=0.2 \mathrm{~mm}$ and strip thickness $17.5 \mu \mathrm{m}$.

the resonant surface and bias voltages that will however impact upon FSS operation. As observed in [24], it could be advantageous for improved isolation to exploit the larger impedance presented by inductors as the minimum selfresonant frequency due to the presence of stray reactances can be approached.

The free-standing FSS with the square unit cell shown in Figure 1 has been investigated as a proof of concept. The periodicity of the array is $p=10.2 \mathrm{~mm}$ and the 9fold bifilar spiral is formed by conductor strips with $0.2 \mathrm{~mm}$ width separated by gaps of $0.4 \mathrm{~mm}$. Initial assessment of the operation of this bistate interwoven spiral AFSS has been performed by CST MWS assuming ideal electrical components. Specifically, the pin diodes across the breaks in the spiral conductor arms are represented as short or open circuits, depending whether they are in the on or off state, respectively, and the inductances as open circuits, without additional stray reactances or losses associated with these lump components. The spiral array has been modeled using a single unit cell with doubly periodic boundary conditions.

The transmittance and reflectance at normal incidence are plotted in Figure 2(a) for the two orthogonal polarizations of the incident field when the pin diodes are in the on state; that is, the breaks in the spiral arms are short circuited, as shown in the inset. For a $y$-polarised (TE) wave, the FSS exhibits pronounced stop-band resonance at about $0.8 \mathrm{GHz}$, which corresponds to the packaging density $\lambda_{r} / p \sim 37\left(\lambda_{r}\right.$ is the resonance wavelength); FBW measured at the $-10 \mathrm{~dB}$ points on the transmission curve is $\sim 63 \%$. For an $x$-polarised (TM) incident wave instead the FSS is practically transparent.

The response of the FSS in the off state, when the pin diodes are considered as open circuits, is shown in Figure 2(b). It can be observed that for the TE wave with the electric field polarization along the $y$-axis, which is affected by the presence of the FSS, the resonance occurs at approximately $1.6 \mathrm{GHz}$ and has narrowband (FBW $\sim 4 \%)$. As a result the transmission loss in the off state is less than $0.5 \mathrm{~dB}$ over the stop-band of the FSS in the on state. The presence of the breaks has practically negligible effect on an incident field polarized along the $x$-axis. The transparency of the FSS in the off state can be further improved by increasing the number of breaks and pin diodes in the spiral arms, which can shift the unwanted FSS resonance upward in frequency. This is shown in Figure 3 for the modified unit cell in the figure inset with a total of four breaks introduced in the spiral arms. It must be noted that while a larger number of breaks and pin diodes can be beneficial for FSS transparency in the off state, they will also cause higher dissipative losses and increase fabrication costs.

\subsection{Effect of Nonideal Switches on the Performance of Bifi-} lar Spiral AFSS. While feasibility of the bistate switching response of the interwoven bifilar spiral elements has been proven, assuming an ideal behaviour of the biasing and switching components, it is important to estimate the impact of imperfect components on the AFSS performance. The main effects of switch parasitics are the dissipative loss and the resonance frequency shift caused by the off state capacitance of switches. To model these effects, we use the simplified equivalent circuits of the forward and reverse biased diodes shown in Figure 4. In the forward bias case (on state), the diode presents a resistance $R_{s}$ in series with the packaging inductance $L_{s}$. At the reverse bias (off state), the circuit becomes a parallel combination of $R_{p}$ with $C_{t}$ in series with $L_{s}$, where $C_{t}$ is actually a combination of the device junction capacitance and its package parasitic.

The main criteria for specification of pin diodes are the size, the cost, and the least forward resistance and off state capacitance. In principle, different technologies are available for the realization of pin diodes with low on state resistance and low off state capacitance. These characteristics however are among the main factors driving the pin diodes cost, and pin diodes with larger off capacitance are generally less expensive. Since in the design of an AFSS a balance has to be struck between the cost of individual devices and the numbers required, for our simulations, we have assumed to use reasonably low cost pin diodes, for example, the device HMSP3862 from Avago [25], which have been recently employed also in [24]. The values used for forward bias are $R_{s}=3 \Omega$ and $L_{s}=0.3 \mathrm{nH}$, while for reverse bias $C_{t}=$ $0.15 \mathrm{pF}$ is added to the $R_{s}-L_{s}$ series circuit. The $R_{p}$ resistance in the off state, usually larger than $10 \mathrm{k} \Omega$, can be neglected in the simulations. The inductors that provide a dc path for the pin diode control signal must be suitably chosen for $\mathrm{RF}$ choke to provide the sufficiently high impedance at the design frequency. In the simulations, we have used $100 \mu \mathrm{H}$ 


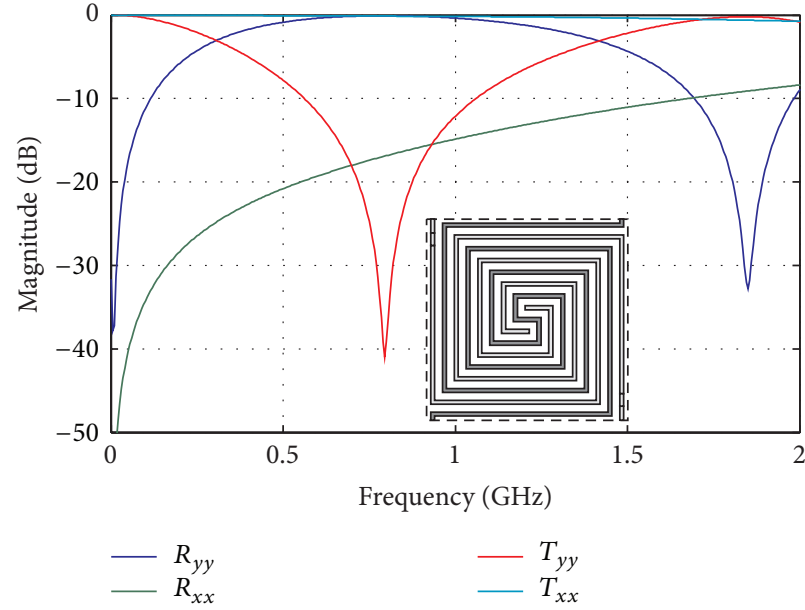

(a)

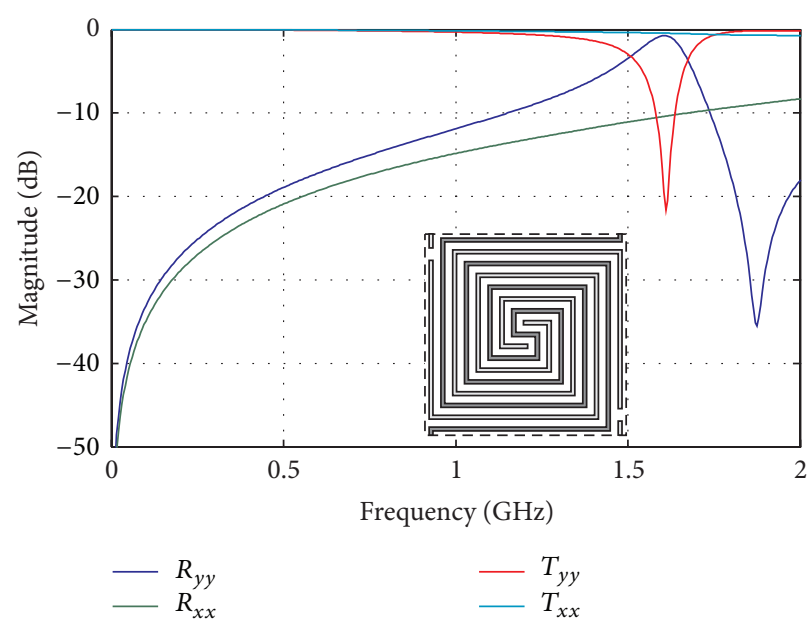

(b)

Figure 2: Transmittance and reflectance of the AFSS with the unit cell of Figure 1 at normal incidence of $x$ - and $y$-polarized waves when ideal pin diode switches are in the (a) on and (b) off states.

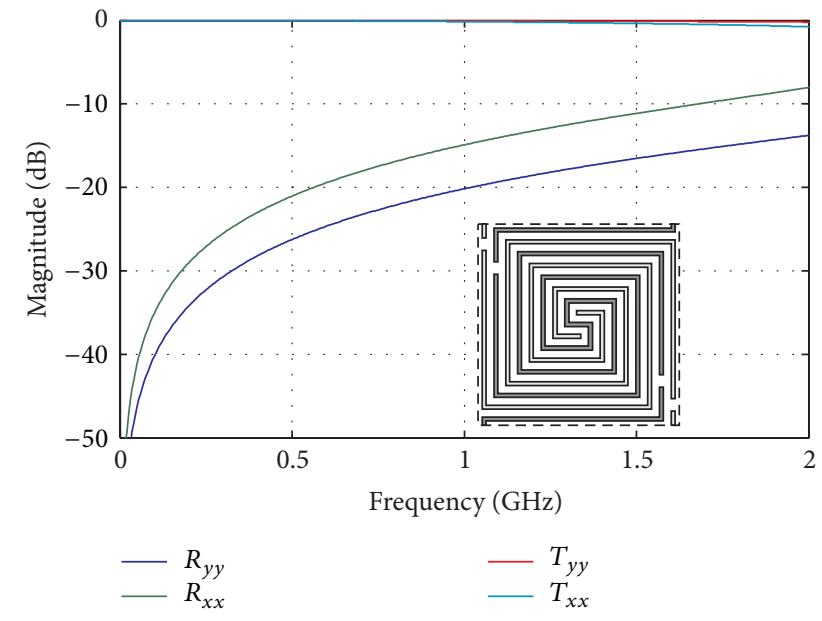

FIGURE 3: Off state transmittance and reflectance of the AFSS of Figure 1 at normal incidence of $x$ - and $y$-polarized waves when two additional breaks and ideal switches are contained in the spiral arms.

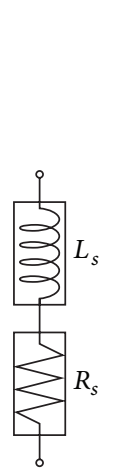

(a)

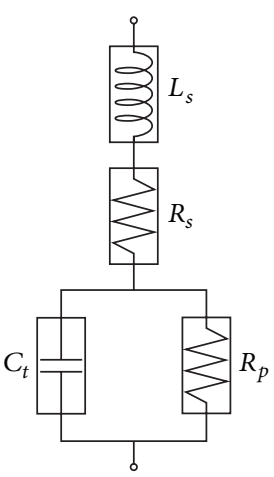

(b)
FIgURe 4: Pin diode equivalent circuits in the (a) forward and (b) reversed bias states.

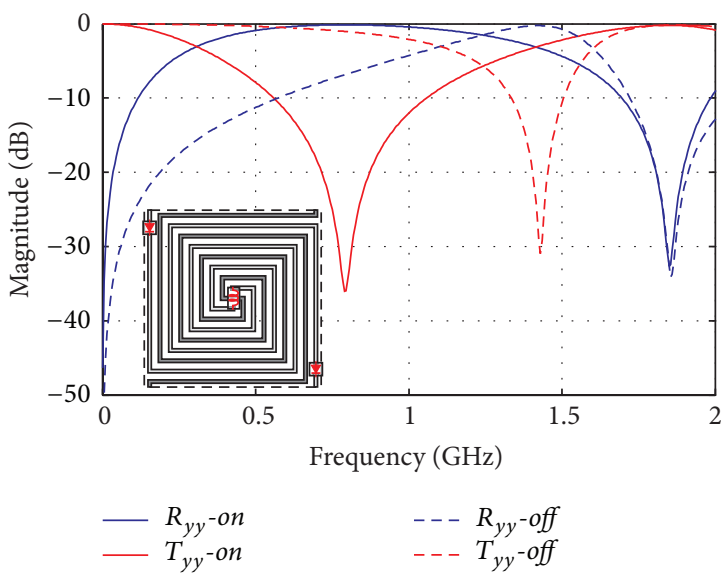

FIGURE 5: Transmittance and reflectance of the AFSS with the unit cell of Figure 1 at normal incidence of a $y$-polarized wave when pin diode switches are in the on (solid lines) and off (dashed lines) states.

inductors available, for example, within the SIMID 1210-100 inductor series from EPCOS [25]. RF choke inductors used in the simulation present a high impedance of approximately $50 \mathrm{k} \Omega$ at the AFSS resonance frequency of $0.8 \mathrm{GHz}$. The $\mathrm{RF}$ choke inductance has been selected by trial and error to minimise distortion of the ideal FSS response (current magnitude across inductors is about $40 \mathrm{dBA}$ smaller than maximum current flowing across conductor strips). Smaller inductance values could be used in practical implementations with just slight degradation of the FSS performance.

The equivalent circuits of the pin diodes and RF choke inductors shown in Figure 4 were incorporated in the CST MWS model of the AFSS shown in Figure 1. Figure 5 displays the simulated transmittance and reflectance of the AFSS illuminated by a normally incident plane wave at both on and off states of the pin diodes. Only the response to the incident field polarized along the $y$-axis is presented because the realistic 


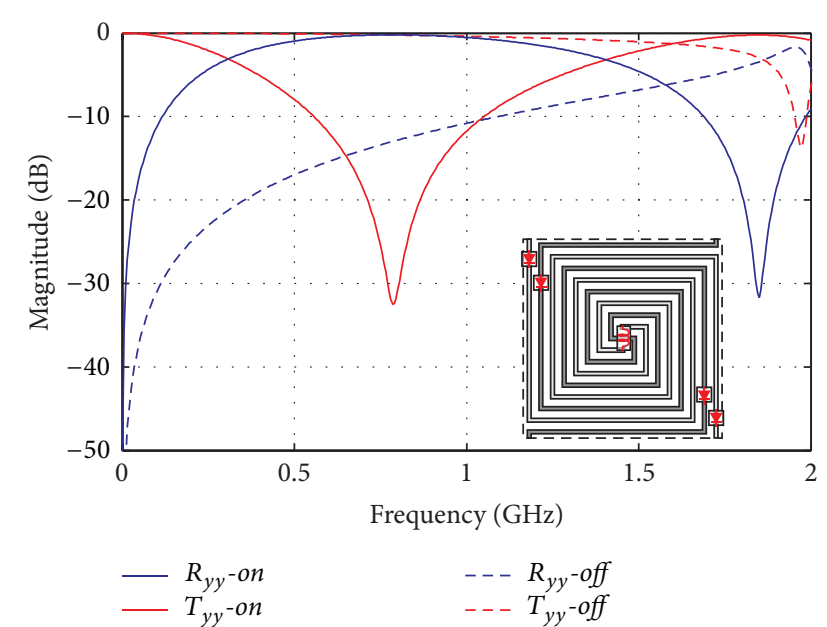

FIGURE 6: Transmittance and reflectance of the AFSS with the unit cell of Figure 1 at normal incidence of a $y$-polarized wave when the four pin diode switches in the spiral arms are in the on (solid lines) and off (dashed lines) states. The on and off states of pin diodes are modelled with their equivalent circuits of Figure 4.

model of pin diodes does not affect the AFSS response at the incident field polarized along the $x$-axis. The transmittance and reflectance in the on state of pin diodes, plotted as solid red and blue lines, respectively, look very similar to the case of ideal switches and absence of the RF choke inductors. Namely, the resonance frequency remains unchanged with the broad stop-band around it, and resonance curve is just slightly shallower than in Figure 2 due to dissipative losses, which also marginally increased FBW (64\%). However, when pin diodes are reverse biased, it is apparent that the off state capacitance of the pin diodes causes shift of the resonance for approximately $0.2 \mathrm{GHz}$ to lower frequency than that in Figure 2(b) of ideal switches. At the same time, the FBW of the secondary stop-band resonance increases from about $4 \%$ to $12 \%$. Both of these factors contribute to reducing the AFSS transparency in the off state as compared to that for ideal switches, with the transmission attenuation in the off state increasing to about $2.6 \mathrm{~dB}$ at the higher frequency edge of the on state reflection band.

To improve the AFSS transparency in the off state, one approach is to increase the number of breaks in the spiral arms that shift the undesired FSS resonance to higher frequencies. This can be achieved by introducing cuts in both the reference spiral and the intertwined spiral arms extended from the surrounding cells, as shown in insert of Figure 3. Figure 6 illustrates the transmittance and reflectance of the AFSS with the unit cell comprising four pin diodes connected across a corresponding number of breaks in the spiral arms, as depicted in the inset. It can be observed that against a small increase of dissipative losses in the on state, in the off state, this surface exhibits good transparency with a transmission loss smaller than $0.5 \mathrm{~dB}$ over the whole stop-band of the surface in the on state.

Instead of increasing their numbers, pin diodes with smaller off capacitance can effectively shift upward the frequency of the parasitic resonance and consequently reduce the off state attenuation. For example, GaAs pin diodes typically have an off capacitance of only $0.036 \mathrm{pF}$ along with a $2.5 \Omega$ forward resistance [26]. Alternatively, RF MEMS switches can provide the key capability of low control power and low insertion loss required in active and reconfigurable FSSs $[27,28]$ and periodic arrays $[29,30]$. The representative values of MEMS off capacitance are in the range $0.05-0.08 \mathrm{pF}$. For example, the AFSS with the layout of Figure 1, using two switches per unit cell with an off capacitance $C_{t}=0.056 \mathrm{pF}$ (either low off capacitance pin diodes or RF MEMS) exhibited the performance similar to that of the AFSS with four low cost pin diodes with $C_{t}=0.15 \mathrm{pF}$.

The transmission and reflection characteristics of the bistate interwoven bifilar spiral AFSS with four pin diodes have been simulated in CST MWS Studio at oblique incidence of TE and TM waves. Similar to the normal incidence, the transmittance and reflectance of TM waves at oblique incidence remain practically indistinguishable in the on and off states of the switches-the FSS is practically transparent and the corresponding plots are not shown. In the case of TE waves, the AFSS in the on state exhibits the high stability of the resonance frequency in a broad range of incidence angles, as illustrated in Figure 7. In the off state, the response is also reasonably stable, with the transmission slowly decreasing with angles of incidence, yet the attenuation remaining below $1 \mathrm{~dB}$ at $45^{\circ}$ oblique incidence. The transparency in the off state slightly decreases at larger incidence angles for the TE polarized waves due to broadening the rejection resonance at about $2 \mathrm{GHz}$. Such resonance broadening is typical for the TE waves incident at slant angles on the interwoven spiral arrays, cf. $[13,31]$.

\section{Dual Polarised Bistate Interwoven Spiral Metasurface}

3.1. Concept and Topology of Dual Polarised AFSS. The concept of interweaving spiral arrays can be further extended to realising switchable isotropic AFSS sensitive to both polarisations of the incident field. Intertwined quadrifilar spirals can be used for this purpose as illustrated by the layout of a $2 \times 2$ cell array depicted in Figure 8 . Similarly to the intertwined bifilar spiral AFSS, a number of strip breaks are cut in the spiral arms at the periphery of the unit cells, and switches are inserted across these breaks. The switch state is controlled by a suitable bias that is supplied by the same conductors forming the spiral arms. The control signal is applied in a grid format, along both rows and columns of the array. It is distributed over the whole surface through the continuous dc current path provided by the pairs of lump inductors at the unit cell centre which act as RF chokes at the design frequency. The inductors are connected between the diagonally opposite open ends of the extended spiral arms and are isolated from each other and the crossing arms of the reference spiral.

The AFSS shown in Figure 8 is isotropic if all the switches are in either on or off states. When the bias is symmetrically applied along both rows and columns of the array to keep all the switches in the on state, the AFSS is reflective in the 


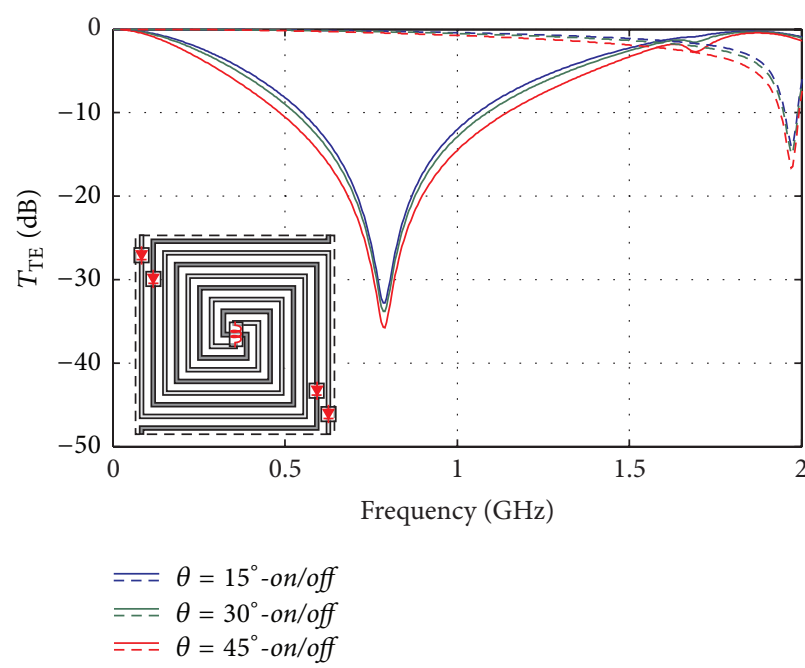

(a)

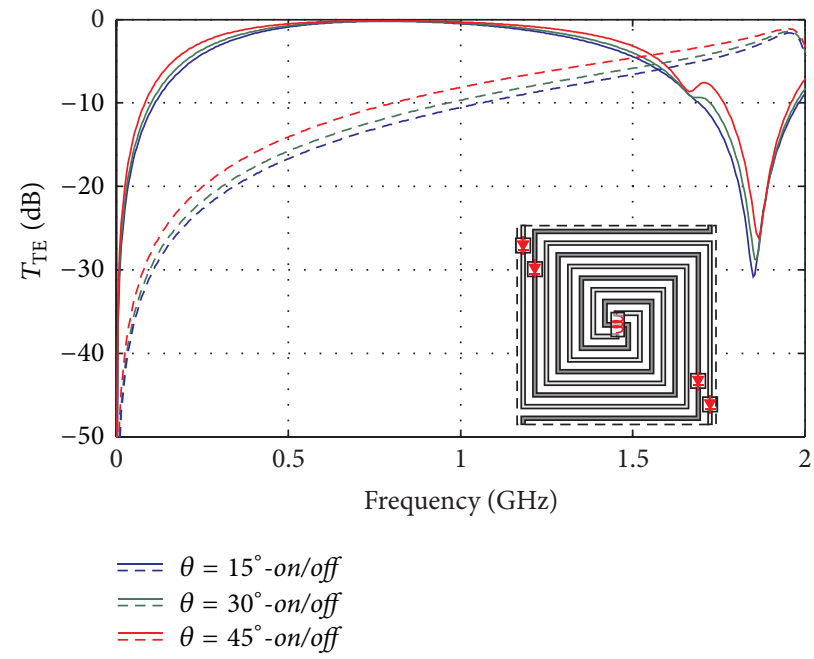

(b)

Figure 7: (a) Transmittance and (b) reflectance of the AFSS with the unit cell of Figure 1, modified with the introduction of two additional pin diode switches, at oblique incidence of TE waves when the pin diodes switches are in the on (solid lines) and off (dashed lines) states. The on and off states of pin diodes are modelled with their equivalent circuits of Figure 4.

specified frequency band and becomes transparent at these frequencies when switches are set in the off state. In the latter mode, the AFSS still exhibits resonance at a higher frequency, which can be placed far enough from the operating band by altering the number of switches inserted in the spiral conductor arms. Dual polarized operation and independent control over orthogonally polarized incident waves can be realised by applying different biases at rows or columns of the array, depending on the targeted polarization sensitivity.

The bistate characteristics of the AFSS depicted in Figure 8 have been simulated in CST MWS assuming first the ideal lossless lumped components, that is, pin diodes modelled as short or open circuits in the on or off state, respectively, and inductances as open circuits. The simulated transmittance and reflectance at normal incidence shown in Figure 9 demonstrate that when the pin diodes are in the on state, the AFSS exhibits a wide rejection resonance at $\sim 0.7 \mathrm{GHz}$, which corresponds to the packaging density $\lambda_{r} / p \sim 42, \mathrm{FBW} \sim 53 \%$ at the transmission level $-10 \mathrm{~dB}$. Conversely, when pin diodes are switched off and act as ideal open circuits, the resonance shifts upward above $2 \mathrm{GHz}$, and the AFSS becomes practically transparent-the transmission loss is less than $0.24 \mathrm{~dB}$ at the high frequency edge of the AFSS stop-band in the on state. The AFSS responses shown in Figure 9 are invariant to the incident field polarization provided that symmetric bias is applied to both rows and columns of the array. The capability of this AFSS for dual polarised operation is illustrated in Figure 10. It is assumed that a nonsymmetric bias voltage scheme is applied to the AFSS to switch on only a pair of pin diodes, whereas the other pair remain in the off state. As a result, the AFSS provides an anisotropic response to the incident field; namely, for a $y$-polarised incident wave, the AFSS exhibits a wide rejection resonance at $\sim 0.7 \mathrm{GHz}$ also seen in Figure 9, while it is practically transparent to the orthogonal polarization along the $x$-axis.
3.2. Effect of Nonideal Switches on the Performance of Quadrifilar Spiral AFSS. The effect of switch parasitics and finite impedance of lumped inductances on the predicted bistate response of the interwoven quadrifilar spiral AFSS needs to be assessed. For this purpose, the equivalent circuits of the pin diodes shown in Figure 4 and the RF choke inductors are incorporated in the analysis of the array structure in Figure 8 in both the forward and reverse bias states. The diode equivalent circuit parameters are the same as in Section 2; that is, for forward bias $R_{s}=3 \mathrm{Ohm}, L_{s}=0.3 \mathrm{nH}$, and for reverse bias, a series capacitance $C_{t}=0.15 \mathrm{pF}$. And a pair of $100 \mu \mathrm{H}$ choke inductors is also included in the unit cell simulation model [25].

The simulated transmittance and reflectance of the AFSS with nonideal lumped components illuminated at normal incidence are shown in Figure 11 for both on and off states of the pin diodes. In the on state, the dissipative losses of switches have negligible effect on the AFSS response which has only slightly shorter stop-band dip and FBW increased to $56 \%$. The effect of the off state capacitance of pin diodes at the reverse bias is more pronounced and causes downward shift of the secondary resonance for $\sim 0.7 \mathrm{GHz}$ and broadening its bandwidth as compared with the ideal case in Figure 9. As a result, the AFSS transparency in the off state is reduced and transmission loss increases to $\sim 1.3 \mathrm{~dB}$ at the upper frequency edge of the reflection band in the on state.

Similarly to the case of the intertwined bifilar spirals, the transparency of the isotropic AFSS in the off state can be improved by using switches with lower off capacitance or/and increasing the number of breaks in the spiral arms of both the reference spiral and the spirals extended from the surrounding cells. This effect is illustrated in Figure 12 which shows the transmittance and reflectance at normal incidence on the modified AFSS with four additional pin diode switches connected across a corresponding number of breaks in the spiral arms, as depicted in the inset of Figure 12, at both on and 


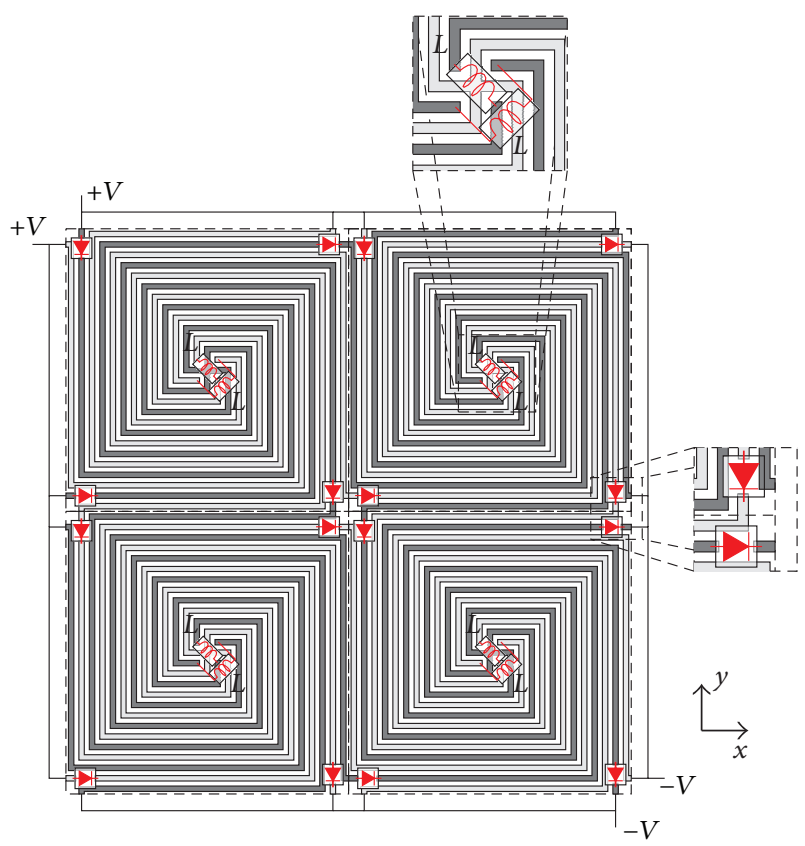

Figure 8: Layout of $2 \times 2$ array portion of sample free-standing bistate interwoven 7 -fold quadrifilar spiral elements. The reference basic spirals in adjacent unit cells are alternatively marked dark and light grey to highlight the intertwining scheme. Four pin diodes are connected across breaks in the outer folds of the spiral conductors and the RF choke inductances are placed at the centre of each unit cell between the open ends of the spiral arms extended from adjacent unit cells. Geometrical parameters of the unit cell: lattice period $p=10.2 \mathrm{~mm}$, spiral pitch $1.6 \mathrm{~mm}$, and strip width $s=0.2 \mathrm{~mm}$ and thickness $17.5 \mu \mathrm{m}$.

off states of the pin diodes. It is noteworthy that transmission loss of this surface in the off state is less than $0.5 \mathrm{~dB}$ over the entire operating band, though improved transparency is obtained at the cost of a small increase of dissipative losses in the on state.

For comparison, Figure 13 shows the improved transparency of the AFSS in the off state that can be obtained with only four switches with the smaller off capacitance $C_{t}=$ $0.056 \mathrm{pF}$. This performance is very similar to that achievable by using eight low cost pin diodes switches with the larger off capacitance $C_{t}=0.15 \mathrm{pF}$. It is necessary to note that the performance of these AFSSs in the off state can also be adjusted by altering the positions of the spiral arm breaks. Identification of the optimal locations of switches is therefore a part of the design process.

The response of the bistate dual polarised AFSSs at oblique incidence of TE and TM waves has been simulated for the unit cell configuration with eight pin diodes shown in insert of Figure 12. The transmittance and reflectance characteristics are displayed in Figure 14 at variable incidence angles of TE and TM waves for both the on and off states of the switchable diodes. These plots demonstrate that, in the on state, the AFSS exhibits high stability of the resonance frequency in a broad range of incidence angles at both TE and TM wave polarisations. The resonance response remains fairly stable also in the off state. The transparency of the

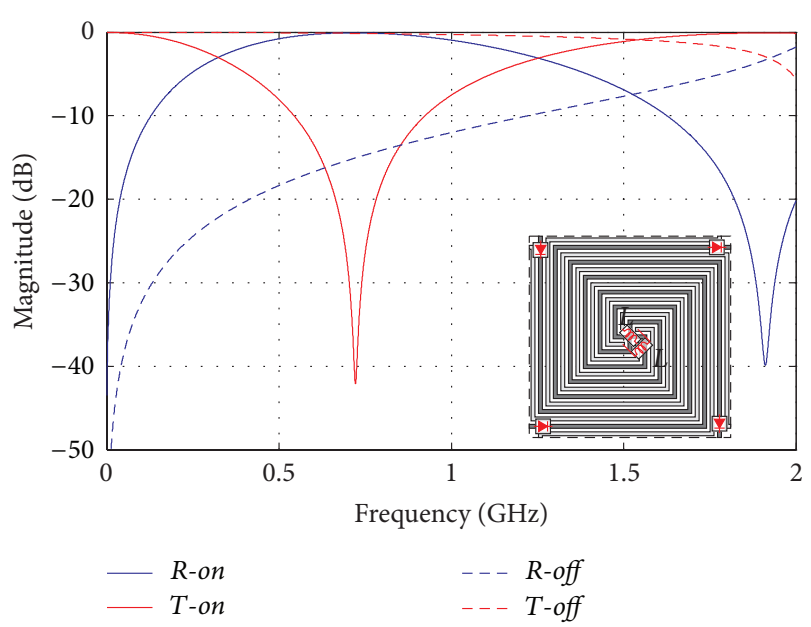

FIGURE 9: Transmittance and reflectance at normal incidence of the AFSS with the unit cell of Figure 8 when ideal pin diode switches are in the on and off states.

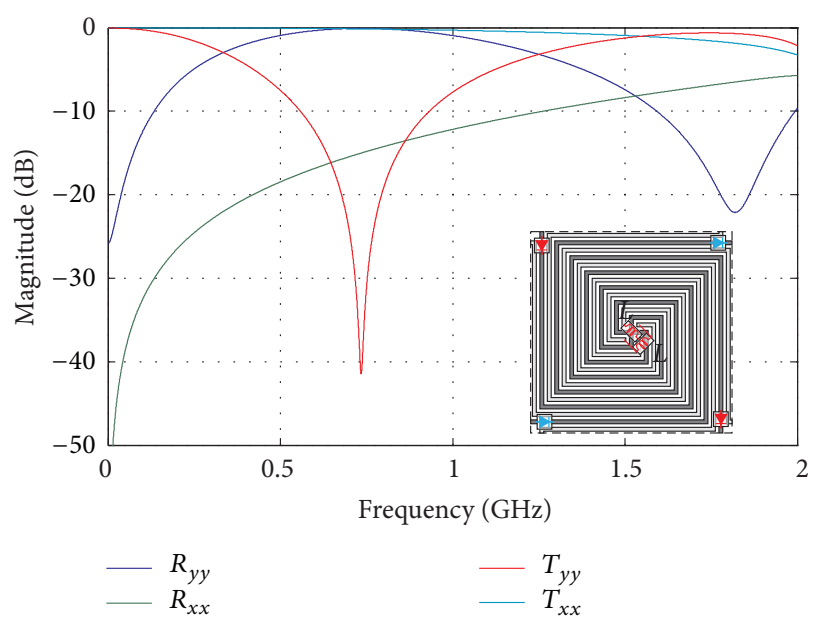

FIGURE 10: Transmittance and reflectance at normal incidence of the AFSS with the unit cell of Figure 8 illuminated by two orthogonal polarizations when a pair of ideal pin diode switches (depicted in red) are in the on state and the other pair (depicted in blue) is in the off state.

surface in the off state slightly decreases at larger angles of incidence of TE polarised waves, whereas it even improves for obliquely incident TM polarised waves. Similarly to the response of bifilar spiral AFSSs at oblique incidence of TE waves, the transparency of quadrifilar spiral AFSSs in the off state is affected by broadening (TE waves) or narrowing (TM waves) of the bandwidth of the rejection resonance at about $2 \mathrm{GHz}$. Such resonance broadening for TE waves and narrowing for TM waves incident at slant angles on the interwoven spiral arrays are the inherent property of this type of FSS, cf. $[13,31]$. At any rate, for both polarizations, transmission losses are found to be less than $1 \mathrm{~dB}$ at $45^{\circ}$ oblique incidence at the upper frequency edge of the reflection band in the on state. 


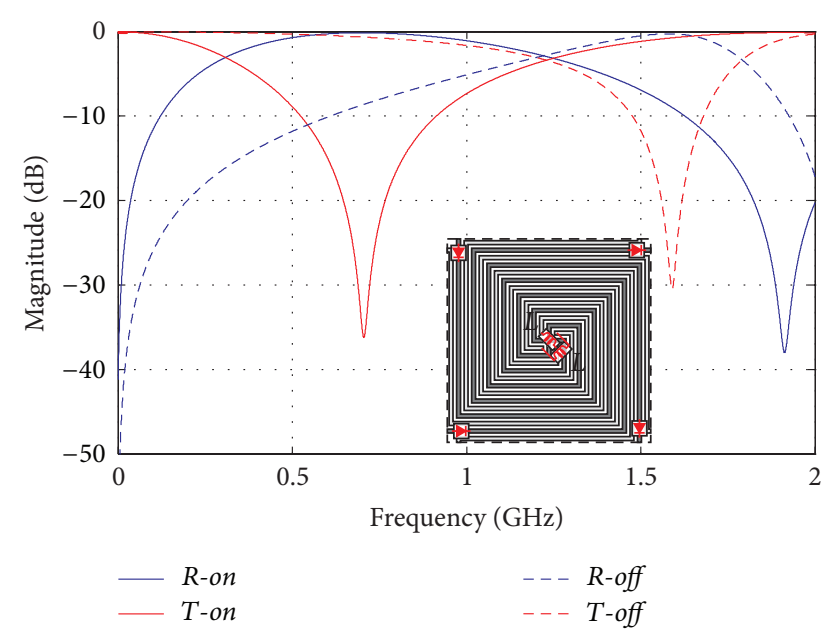

FIGURE 11: Transmittance and reflectance at normal incidence of the AFSS with the unit cell of Figure 8 when pin diode switches are in the on (solid lines) and off (dashed lines) states.

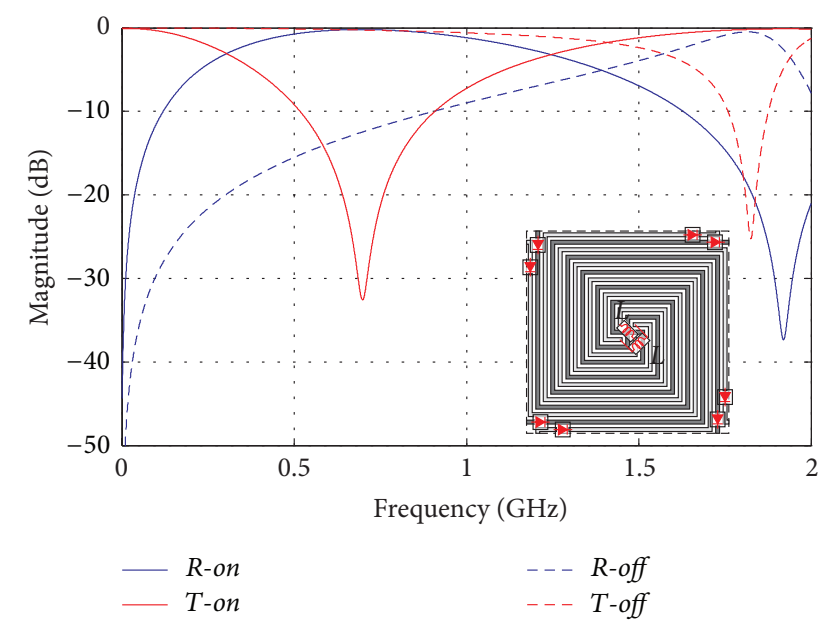

FIGURE 12: Transmittance and reflectance at normal incidence of the AFSS with the unit cell of Figure 8, with four additional pin diode switches introducing the spiral arms, in the on (solid lines) and off (dashed lines) states.

\section{Conclusion}

The bistate voltage-controlled metasurfaces composed of the interwoven spiral arrays with integrated pin diodes are proposed for both single and dual polarisation operation. The primary feature of the presented architecture is the dual use of the spiral conductors as the constituent elements of the AFSS and as the path for supplying dc bias to pin diodes. It has been demonstrated that switching pin diodes between their on and off states enables toggling the AFSS response between the transmission and reflection modes at the specified frequency. Integration of the biasing circuitry with the metasurface conductor pattern has enabled us to eliminate the need of separate wires supplying bias voltage to each switchable diode. The proposed metasurface architecture has dramatically simplified the AFSS topology where

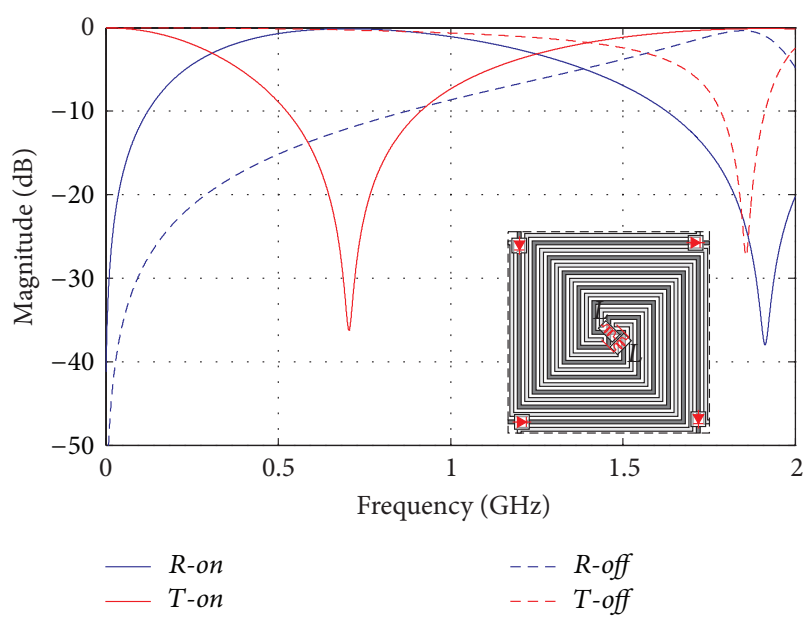

FIGURE 13: Transmittance and reflectance of the AFSS with the unit cell of Figure 8 at normal incidence when switches, with low off capacitance $C_{t}=0.056 \mathrm{pF}$, are in the on (solid lines) and off (dashed lines) states.

small parasitic reactances have minor effect on the array RF response in both on and off states as demonstrated by the presented simulation results.

The detailed analysis has shown that parasitic reactances of the switches strongly influence the overall response of the AFSS, thus making it progressively more difficult to achieve the desired off state transparency at the upper edge of the operational band. Nonetheless, the AFSS transparency in the off state can be improved by increasing the number of switches in the spiral arms, but this inflicts small increase of dissipative losses in the on state. Alternatively, pin diodes with smaller off capacitance can be used for this purpose for expense of higher cost.

It has been demonstrated that the presented AFSS configurations can achieve good isolation between transmission and reflection states over a broad FBW, while retaining the substantial subwavelength response of the original passive metasurfaces. The latter property enables high polarization and angular stability of AFSS. Also owing to the extremely small subwavelength size of their unit cell, the interwoven multifilar spirals are particularly suitable for the design of reconfigurable homogeneous metasurfaces.

\section{Conflict of Interests}

The authors declare that there is no conflict of interests regarding the publication of this paper.

\section{Acknowledgment}

This work was supported by the European Commission 7th Framework Program Marie Curie IAPP Project Grant no. 286333, "Wireless Friendly Energy Efficient Buildings (WiFEEB)." 


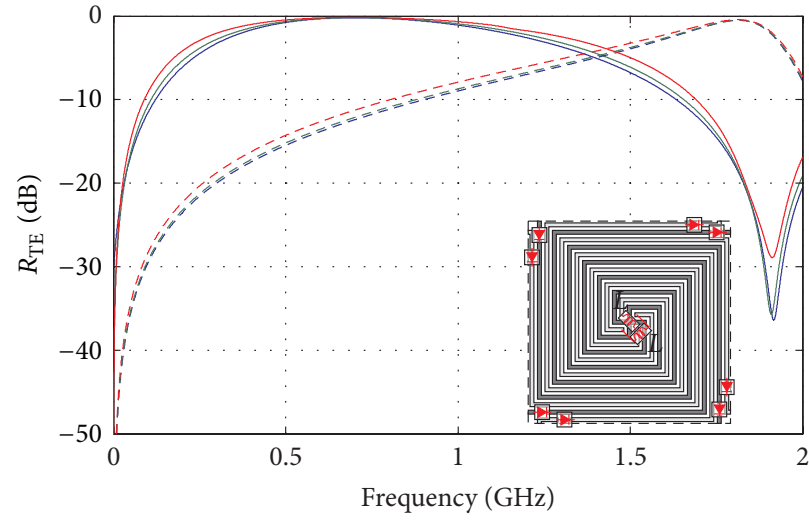

(a)

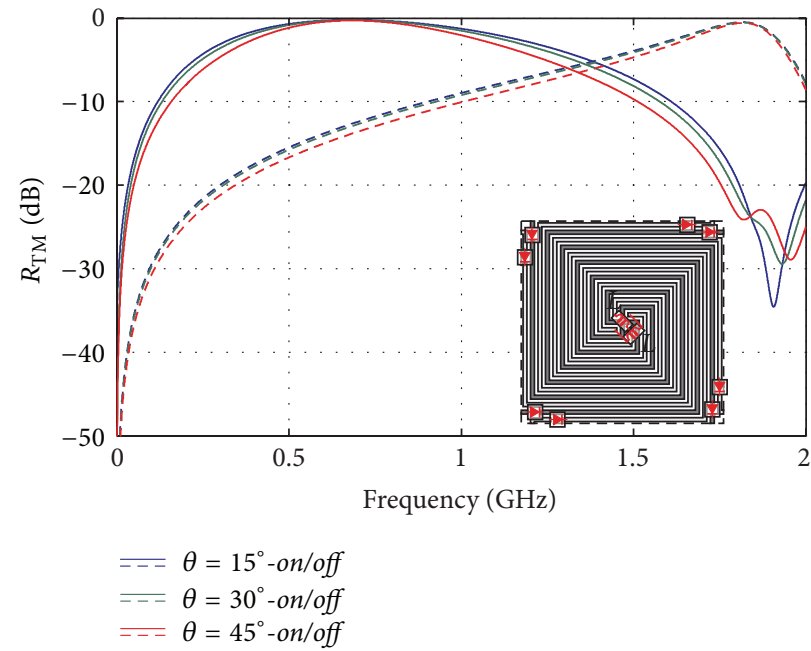

(c)

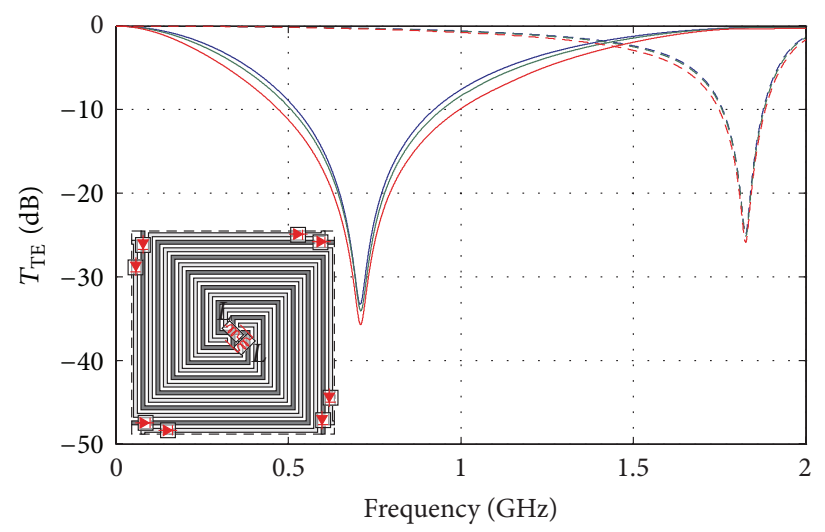

(b)

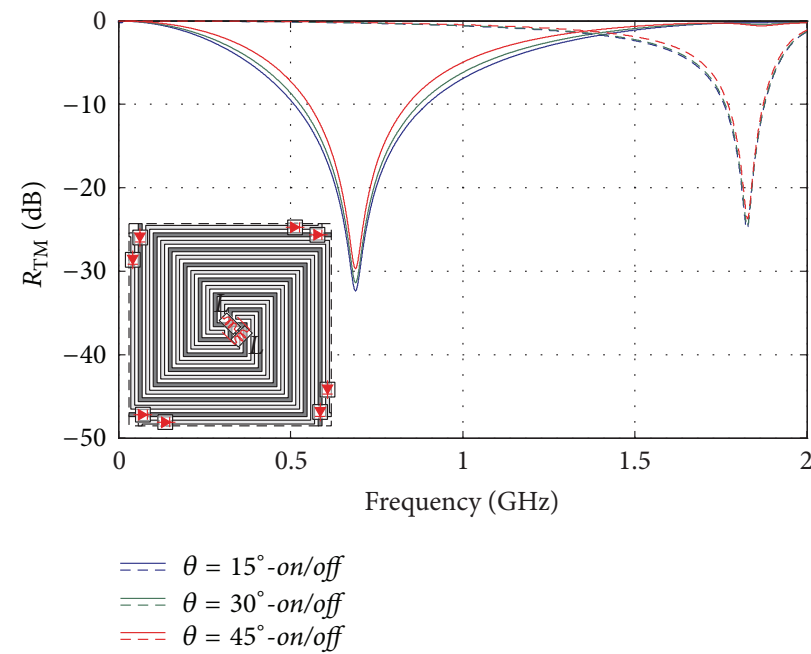

(d)

FIGURE 14: Transmittance and reflectance of the AFSS with the unit cell layout of Figure 8, modified with the introduction of four additional switches, at oblique incidence of TE (a)-(b) and TM (c)-(d) waves when the pin diode switches are in the on (solid lines) and off (dashed lines) states.

\section{References}

[1] C. L. Holloway, E. F. Kuester, J. A. Gordon, J. O’Hara, J. Booth, and D. R. Smith, "An overview of the theory and applications of metasurfaces: the two-dimensional equivalents of metamaterials," IEEE Antennas and Propagation Magazine, vol. 54, no. 2, pp. 10-35, 2012.

[2] Y. Ra'di, V. S. Asadchy, and S. A. Tretyakov, "Total absorption of electromagnetic waves in ultimately thin layers," IEEE Transactions on Antennas and Propagation, vol. 61, no. 9, pp. 4606-4614, 2013.

[3] F. C. Seman and R. Cahill, "Performance enhancement of Salisbury screen absorber using resistively loaded spiral FSS," Microwave and Optical Technology Letters, vol. 53, no. 7, pp. 1538-1541, 2011.

[4] N. I. Landy, S. Sajuyigbe, J. J. Mock, D. R. Smith, and W. J. Padilla, "Perfect metamaterial absorber," Physical Review Letters, vol. 100, no. 20, Article ID 207402, 2008.

[5] N. Papasimakis, V. A. Fedotov, N. I. Zheludev, and S. L. Prosvirnin, "Metamaterial analog of electromagnetically induced transparency," Physical Review Letters, vol. 101, no. 25, Article ID 253903, 2008.
[6] P. Y. Chen and A. Alù, "Mantle cloaking using thin patterned metasurfaces," Physical Review B, vol. 84, Article ID 205110, 2011.

[7] K. Sarabandi and N. Behdad, "A frequency selective surface with miniaturized elements," IEEE Transactions on Antennas and Propagation, vol. 55, no. 5, pp. 1239-1245, 2007.

[8] A. C. M. Austin, M. J. Neve, and G. B. Rowe, "Modeling propagation in multifloor buildings using the FDTD method," IEEE Transactions on Antennas and Propagation, vol. 59, no. 11, pp. 4239-4246, 2011.

[9] F. Huang, J. C. Batchelor, and E. A. Parker, "Interwoven convoluted element frequency selective services with wide bandwidths," Electronics Letters, vol. 42, no. 14, pp. 788-790, 2006.

[10] A. Monorchio, S. Genovesi, E. Carrubba, and G. Manara, "Design of printed FSS for compact and bandwidth enhanced metasurfaces," in Proceedings of the 1st International Congress on Advanced Electromagnetic Materials in Microwaves and Optics, pp. 811-814, 2007.

[11] B. Sanz-Izquierdo, E. A. Parker, J. B. Robertson, and J. C. Batchelor, "Singly and dual polarized convoluted frequency selective structures," IEEE Transactions on Antennas and Propagation, vol. 58, no. 3, pp. 690-696, 2010. 
[12] A. Vallecchi and A. G. Schuchinsky, "Entwined spirals for ultra compact wideband frequency selective surfaces," in Proceedings of the 4th European Conference on Antennas and Propagation (EuCAP '10), pp. 12-16, Barcelona, Spain, April 2010.

[13] A. Vallecchi and A. G. Schuchinsky, "Entwined planar spirals for artificial surfaces," IEEE Antennas and Wireless Propagation Letters, vol. 9, pp. 994-997, 2010.

[14] Z. Bayraktar, J. P. Turpin, and D. H. Werner, "Nature-inspired optimization of high-impedance metasurfaces with ultrasmall interwoven unit cells," IEEE Antennas and Wireless Propagation Letters, vol. 10, pp. 1563-1566, 2011.

[15] E. A. Parker and A. N. A. El Sheikh, "Convoluted array elements and reduced size unit cells for frequency-selective surfaces," IEE Proceedings H, vol. 138, no. 1, pp. 19-22, 1991.

[16] E. A. Parker, A. N. A. El Sheikh, and A. C. C. Lima, "Convoluted frequency-selective array elements derived from linear and crossed dipoles," IEE Proceedings H, vol. 140, no. 5, pp. 378-380, 1993.

[17] T. K. Chang, R. J. Langley, and E. A. Parker, "Active frequency selective surfaces," IEE Proceedings H, vol. 143, no. 1, pp. 62-66, 1996.

[18] A. Vallecchi and A. G. Schuchinsky, "Artificial surfaces formed by tessellations of intertwined spirals," in Proceedings of the 5th European Conference on Antennas and Propagation (EUCAP '11), pp. 1846-1848, Rome, Italy, April 2011.

[19] P. Edenhofer and A. Alpaslan, "Active frequency selective surfaces for antenna applications electronically to control phase distribution and reflective/transmissive amplification," in Proceedings of the IEEE/ACES International Conference on Wireless Communications and Applied Computational Electromagnetics, pp. 237-240, April 2005.

[20] B. M. Cahill and E. A. Parker, "Field switching in an enclosure with active FSS screen," Electronics Letters, vol. 37, no. 4, pp. 244245, 2001.

[21] A. Tennant and B. Chambers, "A single-layer tuneable microwave absorber using an active FSS," IEEE Microwave and Wireless Components Letters, vol. 14, no. 1, pp. 46-47, 2004.

[22] G. I. Kiani, K. L. Ford, K. P. Esselle, A. R. Weily, C. Panagamuwa, and J. C. Batchelor, "Single-layer bandpass actlve frequency selective surface," Microwave and Optical Technology Letters, vol. 50, no. 8, pp. 2149-2151, 2008.

[23] G. I. Kiani, K. L. Ford, L. G. Olsson, K. P. Esselle, and C. J. Panagamuwa, "Switchable frequency selective surface for reconfigurable electromagnetic architecture of buildings," IEEE Transactions on Antennas and Propagation, vol. 58, no. 2, pp. 581-584, 2010.

[24] P. S. Taylor, E. A. Parker, and J. C. Batchelor, "An active annular ring frequency selective surface," IEEE Transactions on Antennas and Propagation, vol. 59, no. 9, pp. 3265-3271, 2011.

[25] http://www.avagotech.com/pages/en/rf_microwave/diodes/pin/ hsmp-3862.

[26] K. W. Kobayashi, A. K. Oki, D. K. Umemoto, S. Claxton, and D. C. Streit, "GaAs HBT PIN diode attenuators and switches," in Proceedings of the IEEE Microwave Millimeter-Wave Monolithic Circuits Symposium Digest, pp. 349-352, Atlanta, Ga, USA, June 1993.

[27] B. Schoenlinner, A. Abbaspour-Tamijani, L. C. Kempel, and G. M. Rebeiz, "Switchable low-loss RF MEMS Ka-band frequencyselective surface," IEEE Transactions on Microwave Theory and Techniques, vol. 52, no. 11, pp. 2474-2481, 2004.
[28] G. M. Coutts, R. R. Mansour, and S. K. Chaudhuri, "Microelectromechanical systems tunable frequency-selective surfaces and electromagnetic-bandgap structures on rigid-flex substrates," IEEE Transactions on Microwave Theory and Techniques, vol. 56, no. 7, pp. 1737-1746, 2008.

[29] Y. Raedi, S. Nikmehr, and A. Poorziad, "A novel bandwidth enhancement technique for X-band RF mems actuated reconfig-urable reflectarray," Progress in Electromagnetics Research, vol. 111, pp. 179-196, 2011.

[30] F. A. Tahir, H. Aubert, and E. Girard, "Equivalent electrical circuit for designing mems-controlled reflectarray phase shifters," Progress in Electromagnetics Research, vol. 100, pp. 1-12, 2010.

[31] A. Vallecchi and A. G. Schuchinsky, "Artificial surfaces of intertwined square spirals: a CPW model," in Proceedings of the IEEE International Microwave Symposium Digest, pp. 1-3, Montreal, Canada, June 2012. 

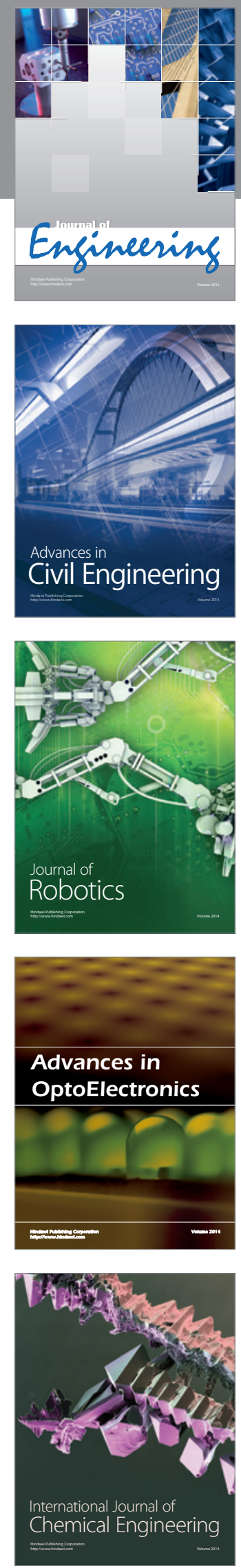

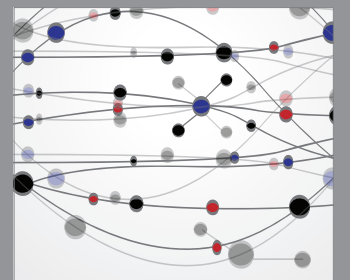

The Scientific World Journal
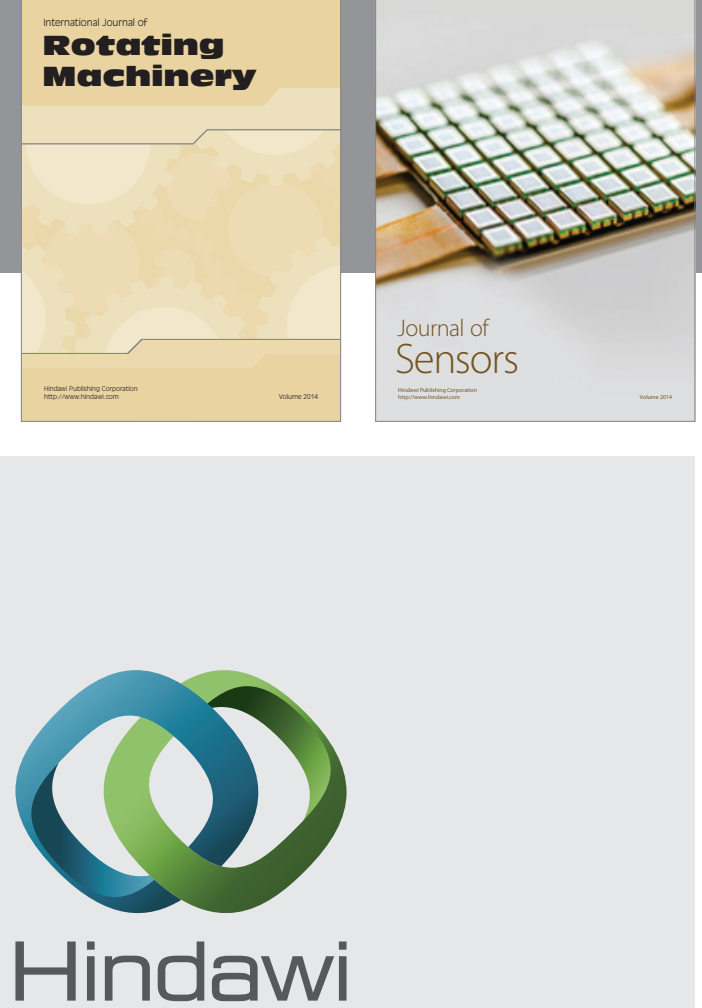

Submit your manuscripts at http://www.hindawi.com
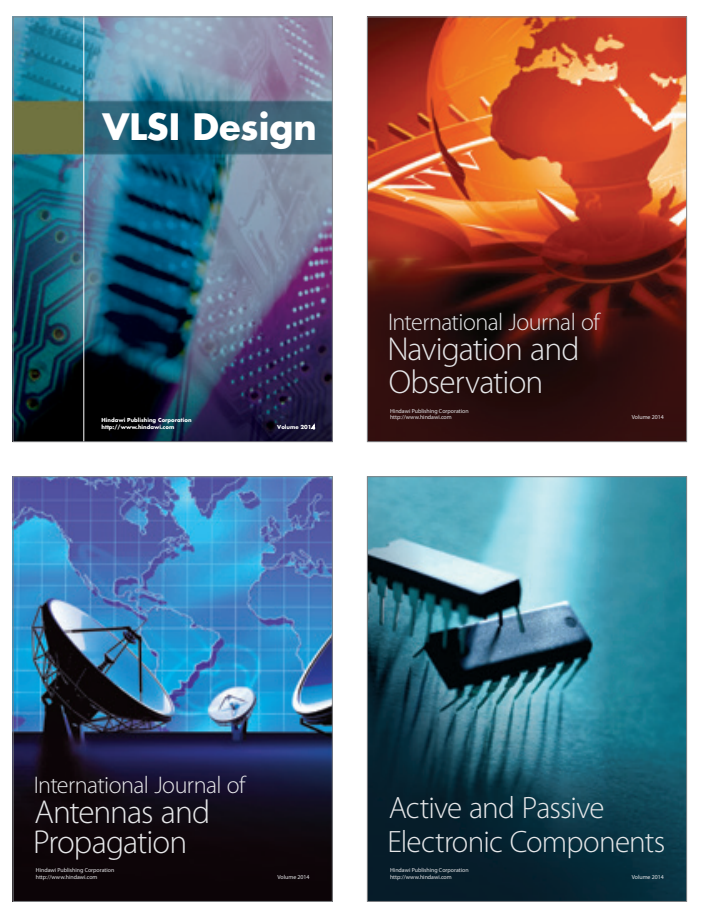
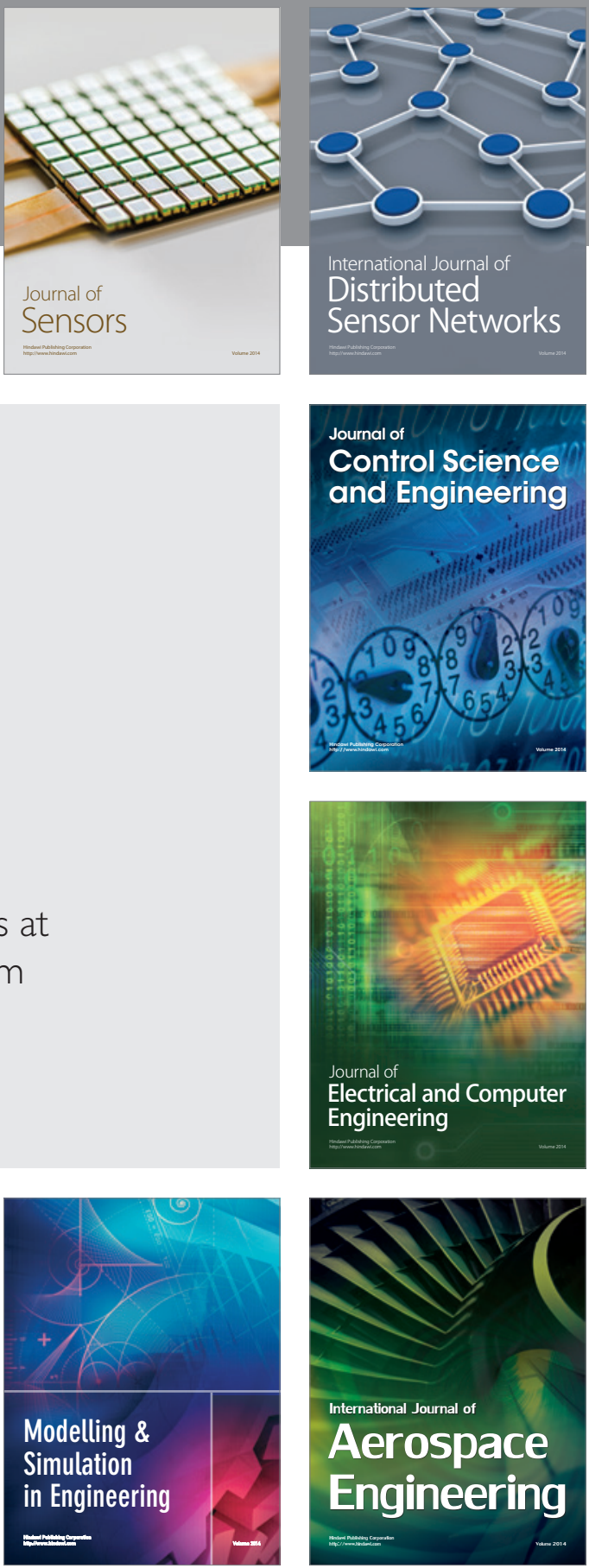

Journal of

Control Science

and Engineering
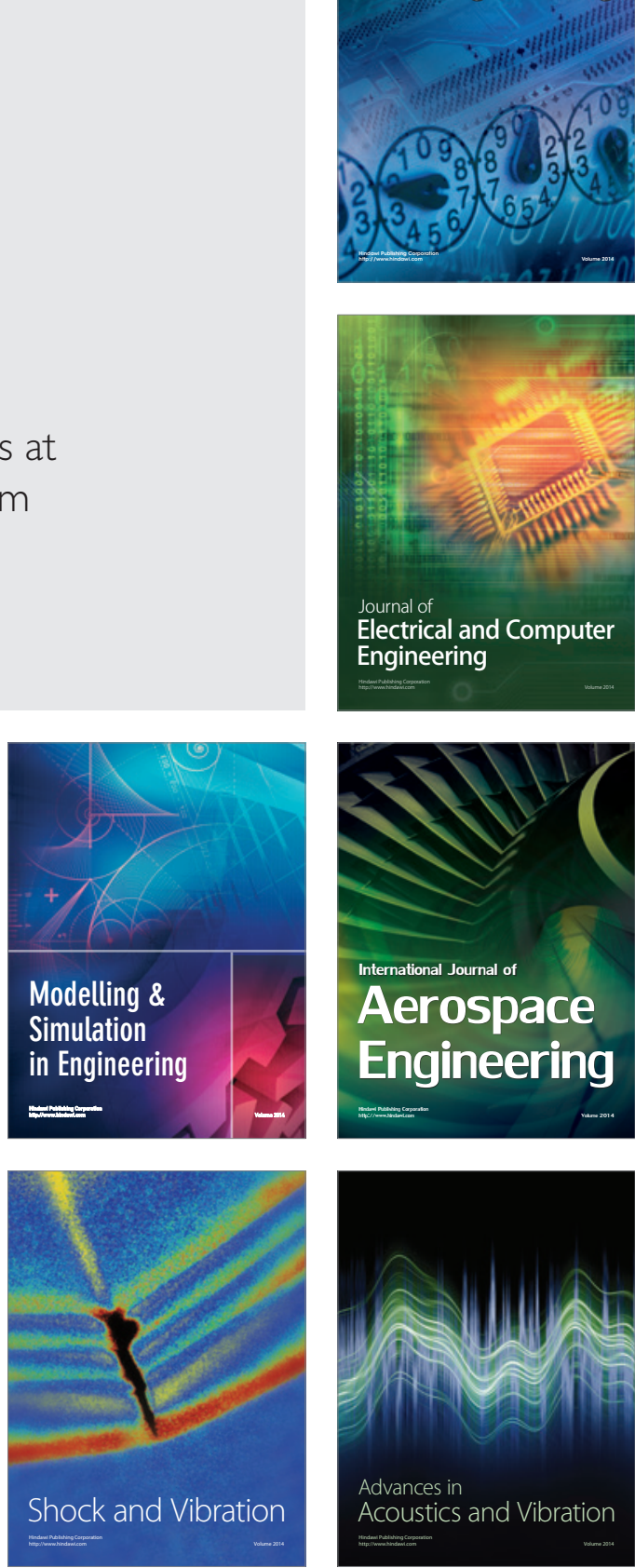